\title{
A Case Of Splenic Artery Aneurysm, Treated By Aneurysmectomy And Splenic Artery End-To-End Anastomosis
}

\author{
Mohammad Alşalaldeh* and Bilgin Emrecan \\ Department of Cardiovascular Surgery Pamukkale University, Faculty of Medicine Hospital, Denizli/ Turkey
}

*Corresponding author: Mohammad Alşalaldeh, Department of Cardiovascular Surgery Pamukkale University, Faculty of Medicine Hospital, Denizli/ Turkey.

\begin{abstract}
We are reporting a case of 70 year old male patient, presented with chief complaint of mid and upper abdominal pain and discomfort for about 3 months and contipation for several days, investigations showed a splenic artery aneurysm of about $4 \mathrm{~cm}$, patient was treated by open surgery and aneurysmectomy was done followed by splenic artery end-to-end anastomosis without touching the spleen. Patient was followed up in our intensive care unit (ICU) for one day then transferred to the floor. On the 6. day of the operation he was discharged with no complaint.
\end{abstract}

Keywords: Abdominal Pain; Splenic Artery Aneurysm (SAA); Open Surgery; Aneurysmectomy; End-To-End Anastomosis

\section{Introduction}

Splenic artery aneurysms (SAA)'s account about $60 \%$ of all splanchnic artery aneurysms and are the third common intrabdominal aneurysm after abdominal aorta and iliac artery aneurysms [1]. SAA's are important because of their rupture potential and may cause fatal haemorrhagia, mainly when the aneurysm's diameter is more than $8 \mathrm{~cm}$ [2]. SAA can be treated either by conventional open surgery or by endovascular intervention techniques. İn our case, we could'nt do endovascular interventional treatment because of the patient's insurance, which does'nt accept such treatment types. So we operated our patient via laparotomy where aneurysmectomy followed by splenic artery end-to-end anastomosis were done, spleen was preserved and was not touched or removed.

\section{Case Report}

A 70 year old male patient, presented to an outer medical center complaining of moderete to severe mid and upper abdominal pain for about 3 months and constipation for several days. He was admitted there as a case of suspected intestinal obstruction and investigations were done, CT-Scan with contrast showed a splenic artery aneurysm of about 37x45 mm (Figure 1). He wa treated there for constipation as a simple case without intestinal obstruction but the patient continued complaining from abdominal pain. Thus he was transferred to our center for further invetstigations and treatment. Here, after examining the patient and evaluate the CT-Scan and because the patient is symptomatic and the diameter of the aneurysm carries a high risk of rupture, operation decision was taken and the pre operation preperations were started. On the operation day, the patient underwent surgery under general anesthesia with a nasogastric tupe insertion, upper mid laparotomy was performed. The İntestine and the stomach were retracted laterally far away from left upper quadrant where the aneurysm of the splenic artery was plapated first then dissected from the head of the pancreas and the surrounded tissues which were adherent to strongly (Figure 2). Our plan was to to preserve the spleen as possible as we can and kept the plan of splenectomy as the last choice if bleeding or possible injury to the pancreas would be detected. After the aneurysm had been dissected from the nearby tissues, heparin was given İ.V in a standard dose, after applying the vascular clamps to pre and post aneurysmatic splenic artery, the aneyreysmatic segment was resected. End-toend anastomosis was performed to the both edges of the splenic artery by using 6.0 prolene suture (Figure 3a). Haemostasis was secured and one abdominal dain was inserted up to the site of the 
anastomosiz. Abdominal wall was closed in layers and the patient was sent to the intensive care unite (ICU) after extubation. He kept in the ICU for one day where the total drainage was about $50 \mathrm{ml}$. On the first day postoperativelly he was transferred to the floor where daily dressing and investigatins were done, medical treatmen was given. On the 6. day of the operation the patient was discharged in good health with no complaint to be followed up as an out patient.

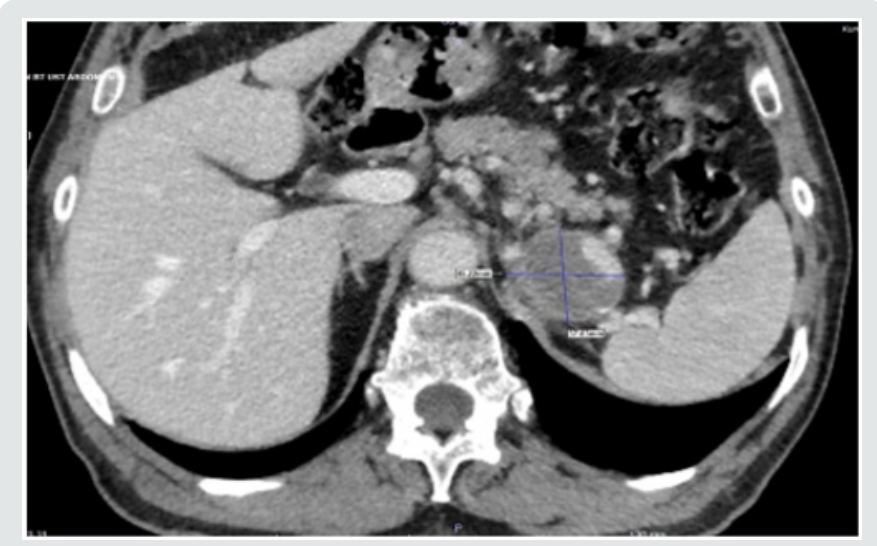

Figure 1: Abdominal CT-Scan with contrast showing a large splenic artery aneurysm of about $37.6 \times 45.2 \mathrm{~mm}$.

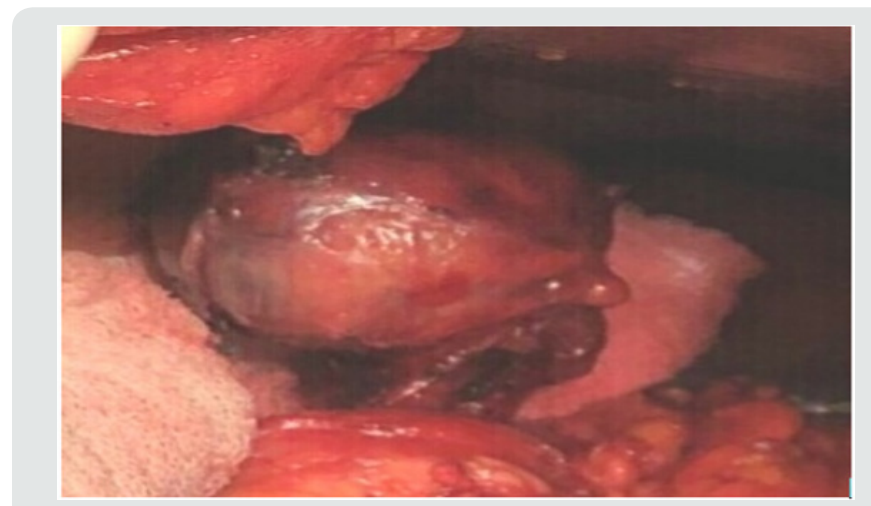

Figure 2: SAA after had been dissected from the surrounding tissues and from the head of the pancreas.

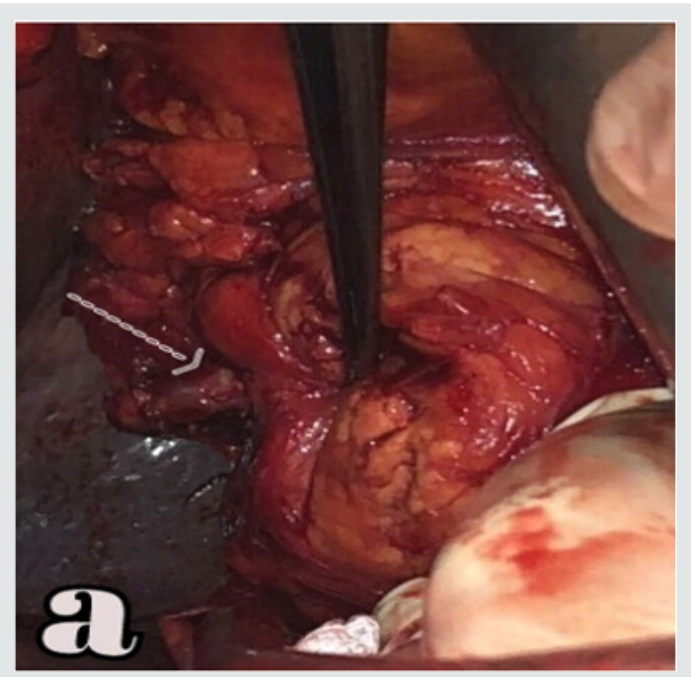

Figure 3a: end-to-end anastomosis of the splenic artery after the aneurysm had been resected.

\section{Discussion}

Splenic artery aneurysms (SAA)'s, are considered the third common intrabdominal aneurysms after abdominal aorta and iliac arteries aneurysms and accounting about $60 \%$ of all splanchnic artery aneurysms. They carry high risk of rupture, mainly when their diameter is large [1,2]. SAA's can be found as true or pseudoaneurysms, as they can rupture intraperitoneally they have the potatial to rupture into the nearby hollow organs or fistulize into pancreatic duct [2]. The incidense of SAA's are about $0.02 \%$ of all age grups with a peak in elders. Most of SAA's are detected accidentlly. Patients may present with non-specific simple abdominal pain and they may be totally asymptomatic [3]. In general, open surgery is a preferable surgical treatment type for the giant splenic artery aneurysms mainly when they are larger than $4 \mathrm{~cm}$ in diameter [4]. Recently endovascular treatment becomes an alternetive treatment type to the conventional open surgery mainlly for the small aneurysms [5]. In some complicated cases mainly when its difficult to reach the aneurysm or when introperative bleeding, nearby organ injuries are occured, or in the cases of aneurysm rupture, the surgeon may have to do splenectomy with total removal of the splenic artery and its aneurysm [6]. In our case the patient was symptomatic and the splenic artery aneurysm was of about $4 \mathrm{~cm}$ in diameter, endovascular coil embolization or stenting was one of the treatment choices, but the health insurance of the patient had not accepted to cover the paymant of of such intervention so the descion was to do open surgery as soon as possible. During the operation we faced difficulty in separeting the aneurysm from the nearby tissues mainly from the head of the pancreas, which was adherent strongly. With patiency, we could dissected the saccular aneurysm from the surrounded tissues without have any injury to the head of pancreas or having any obvious bleeding (Figure $3 \mathrm{~b}$ ). The proximal and distal parts of the splenic artery around the aneurysm were close to each other enough, so we could anastomose them in shape of end-to-end with no need to use graft. Post operativly the patient had only a pain in the site of the operation, drainage was of about $50 \mathrm{ml}$. On the 6 . day of the operation he was discharged with no complaint.

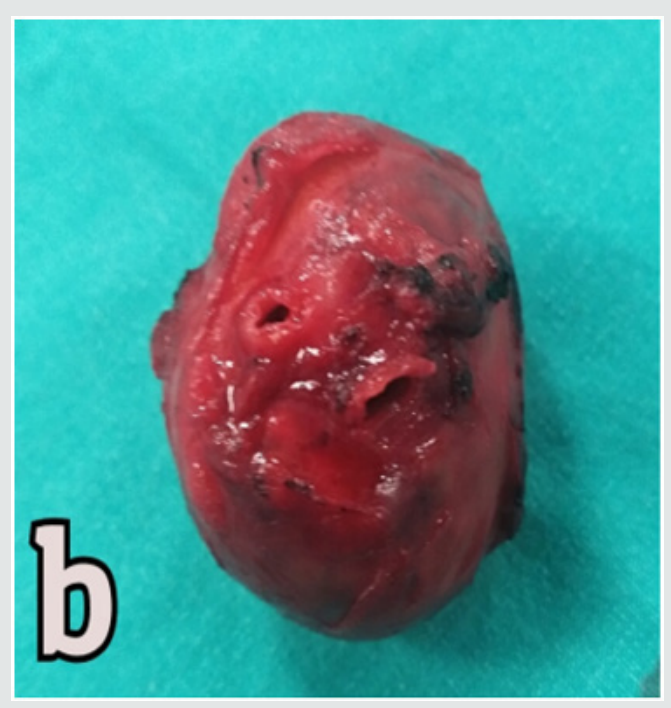

Figure 3b: SAA after resection. 


\section{Result}

SAA's are rare but have high risk of rupture so they should be treated as soon as they are detected. Open surgery is still the standard form of treatment, endovascular intervention is an alternative method of treatment mainly for small SAA's. In large SAA, we prefer to do "spleen preserving open surgery" if it is possible, end-to-end anastomosis of the splenic artery ,after aneurysm resection, can be done easily with no need for graft interposition, the most difficult step of the operation is the dissection of the aneurysm from adherent nearby tissues and pancreas, if you can do it smoothly with patiency you can manage the other steps without complications and with good result after surgery.

\section{Conclusion}

In large SAA's, spleen preserving aneurysmectomy open surgery can be done with cautiousness and patiency and the result of surgery is usually satisfactory.

\section{References}

1. Akbulut S, Otan E (2015) Management of giant splenic artery aneurysm: comprehensive literature review. Medicine 94(27): e1016.

2. Orsitto G, Fulvio F, Pinto AG (2011) Geriatric assessment of a giant splenic artery aneurysm accidentally diagnosed. Aging Clin Exp Res 23: 491-494.

3. Vlychou M, Kokkinis C, Stathopoulou S (2008) Imaging investigation of a giant splenic artery aneurysm. Angiology 59: 503-506.

4. Pulli R, Dorigo W, Troisi N, Pratesi G, Innocenti AA, et al. (2008) Surgical treatment of visceral artery aneurysms: a 25 year experience. J Vasc Surg 48: 334-342.

5. Pulli R, Innocenti AA, Barbanti E (2001) Early and long-term results of surgical treatment of splenic artery aneurysms. Am J urg 182: 520-523.

6. Abdulrahman A, Shabkah A, Hassanain M, Aljiffry M (2014) Ruptured spontaneous splenic artery aneurysm: A case report and review of the literature. International journal of surgery case reports 5(10): 754-757.

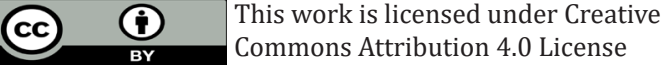

To Submit Your Article Click Here: Submit Article

DOI: $10.32474 /$ RRHOAJ.2019.04.000182

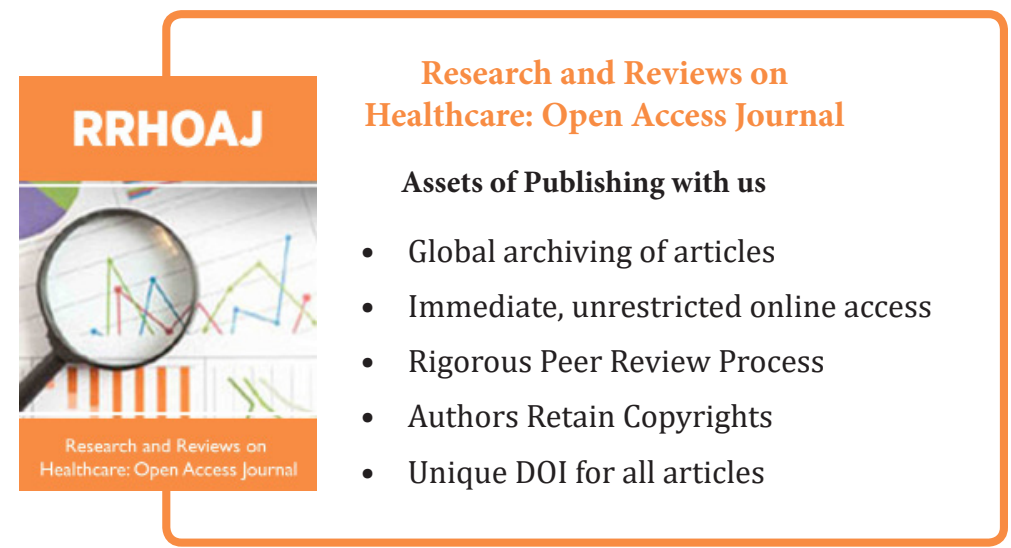

